\title{
Comparative Assessment of White Rust Disease in Improved Varieties of Indian Mustard
}

\author{
Ladhu Ram* and R.P. Awasthi
}

G.B. Pant University of Agriculture and Technology, Pantnagar, Uttrakhand-263145, India

*Corresponding author

\begin{abstract}
A B S T R A C T

\begin{tabular}{|l|}
\hline K e y w o r d s \\
White rust disease, \\
Albugo candida, \\
Brassica juncea, \\
Indian Mustard \\
\hline Article Info \\
\hline $\begin{array}{l}\text { Accepted: } \\
12 \text { April } 2018 \\
\text { Available Online: } \\
\text { 10 May } 2018\end{array}$ \\
\hline
\end{tabular}

Indian mustard (Brassica juncea) is the most important and major edible oil seed crop. White rust [Albugo candida (Pers. Ex.Lev.) Kuntze] is the important disease which can cause major yield losses in Indian Mustard. This study shows comparative assessment of white rust disease index (\%), white rust disease severity and white rust disease incidence on leaves as well as on the inflorescence in the improved varieties of Indian Mustard. The experiment was conducted in RBD in split plot arrangement with three replications. Nine different improved varieties of Indian Mustard (Brassica juncea) were used as main plots, while protected and unprotected treatments were considered in sub-plots separately. Per cent disease index (PDI) of white rust on leaves of different genotypes of Brassica juncea was recorded at four stages of plant growth i.e. 80 DAS, 90 DAS, 100 DAS and 110 DAS while staghead severity and staghead incidence were recorded one week before maturity. Brassica juncea, varieties PR-2006-14 and NDRE-4 found susceptible to white rust which shows maximum PDI (74.97) and 56.31 respectively while varieties PRB-2008-5, PRE2009-12, and PRB-2004-3-4 found slightly tolerant to White rust disease and shows minimum PDI (52.15), (49.98) and (49.98) respectively.
\end{abstract}

\section{Introduction}

Indian mustard is the most important and major edible oil seed crop after soybean. It is a very good source of oil varying from 35-48 per cent which has several anti-nutritional factors like erucic acid (22:1) and eusinic acid (20:1). It is also used as a spice or condiment in preparation, seasoning and stuffing of foods and pickles in India.

Apart from this, its oil is also used for treating medicinal remedies like stomach aches, bone aches, muscle pains, skin disorders etc.
The estimated area, production and productivity of rapeseed-mustard in the world was 30.74 million hectares ( $m$ ha), 59.93 million tonnes $(\mathrm{m} \quad \mathrm{t})$ and $1,950 \mathrm{~kg} / \mathrm{ha}$, respectively, during 2009-10. Globally, India produces 7.565 million tonnes $(\mathrm{m} \mathrm{t})$ from 6.583 million hectares (m ha) with productivity of $11.74 \mathrm{~kg} / \mathrm{ha}$ (GOI, 2011-12).

Despite considerable increase in the productivity and production under Technology Mission, huge amount of money is spent on the import of edible oil. A wide gap exists between the potential yield and the yield 
realized at the farmer's field, which is largely because of number of biotic and abiotic stresses to which the rapeseed-mustard crop is exposed. Major biotic stresses are Alternaria blight disease [Alternaria brassicae (Berk.) Sacc.], white rust [Albugo candida (Lev.) Kuntze], and downy mildew [Hyaloperonospora parasitica (Pers.) ex. Fr.].

Mixed infection of white rust and downy mildew in inflorescence of Brassica crop showed 37-47 per cent less pods with reduction of 17-32 per cent grain yield (Bains and Jhooty, 1979). Petrie and Vanterpool (1974) from Canada reported that systemic infection causes average reduction of 60 percent in seed yield on individual plants. Saharan (1992) reported 23-55 per cent yield loss in B. juncea and Bisht et al., (1994) reported 17-37 per cent yield loss in Rapeseed-Mustard due to mixed infection of white rust and downy mildew. Yield losses ranging between 23 to $54.5 \%$ due to white rust have been reported from India (Saharan et al., 1984). Sangeetha and Siddaramaiah (2007) have reported that a maximum temperature of 26 to $29^{\circ} \mathrm{C}$, a minimum temperature of 14 to $15^{\circ} \mathrm{C}$ and average relative humidity of more than $65 \%$ favour the development of these diseases. The pustules of white rust developed at a faster rate when the average relative humidity was more than $65 \%$ and average temperature was between 10 to $18^{\circ} \mathrm{C}$ (Lakhra and Saharan, 1991).

This study shows comparative assessment of white rust disease severity, incidence and Percent Disease Index (PDI) in improved varieties of Indian Mustard (Brassica juncea) for consecutive two years during 2011-12 and 2012-13.

\section{Materials and Methods}

Field experiment was conducted for consecutive two crop season during 20112012 and 2012-2013 at the NEB Crop
Research Centre, G.B Pant University of Agriculture and Technology, Pantnagarfor comparative assessment of white rust disease severity in the improved varieties of Indian Mustard.

The experiment was conducted in RBD in split plot arrangement with three replications. Nine different improved varieties of Indian Mustard (Brassica juncea) were used as main plots, while protected and unprotected treatments were considered in sub-plots separately. In protected plot alternate spray of fungicides viz. Mancozeb @ $0.2 \%$ and Ridomil MZ @ 0.25\% at weekly intervals were done as much as possible to completely control the diseases. In case of unprotected plot no fungicide was applied as to provide the natural conditions as much as possible to create maximum disease pressure. Mancozeb was used to manage the Alternaria blight disease and also to overcome the development of resistance among the pathogens.

\section{White rust disease severity at leaf stage}

Average disease index on true leaves due to white rust was taken at 80,90,100 and 110 days after sowing (DAS) by using 0-6 disease rating scale (Conn et al., 1990) as follows: Fifteen leaves were randomly collected from each treatment and rated as per the above rating scale and disease index was calculated separately for each treatment. The formula given by Wheeler (1969) was used to calculate the per cent disease index as follows:

$\frac{\text { Disease } \begin{array}{c}\text { index } \\ \text { Sum of all numerical ratings }\end{array}}{\text { No.of leavesexamined }(15) \times \text { Maximum grade of scale }(6)} \times 100$

\section{Staghead incidence}

Total number of plants, and plants affected by staghead symptoms were counted in each treatment. The staghead incidence was calculated with the help of following formula: 
Staghead incidence
$\frac{\text { Number of plants showing staghead }}{\text { Total number of plants }} \times 100$

\section{Staghead severity}

Total racemes of five staghead affected plants and number of racemes showing staghead symptoms per plant was counted and staghead severity was calculated as per the formula given below:

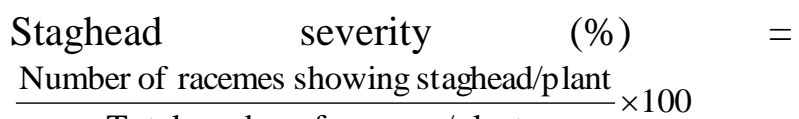

Total number of racemes/plant

\section{Per cent Disease Control (PDC)}

Per cent disease control was calculated at different plant growth stages with the help of following formula:

Per cent disease control $=$

$\frac{\text { Disease incidence or severity in unprotected plot-Disease incidence or severity in protected plot }}{\text { Disease incidence or severity in unprotected plot }} \times 100$

\section{Results and Discussion}

\section{White rust disease index (\%)}

Percent disease index of white rust on leaves of different genotypes of Brassica juncea was recorded at four stages of plant growth i.e. 80 DAS, 90 DAS, 100 DAS and 110 DAS during 2011-12 and 2012-13.

At 80 DAS, maximum white rust index was recorded in genotype PR-2006-14(49.78\%) followed by PRB-2008-5 (47.38\%) and PRE2009-12 (47.38\%) in unprotected plots during 2011-12. Genotype PRB-2008-5, PRE-200912, PRB-2004-3-4, Divya, NDRE-4 and Kranti showed the minimum white rust index on leaf (24.09\%) followed by PRE-2007-6, PR-200813, and PR-2006-14(25.33\%) in protected plots. Similarly NDRE-4 showed the maximum white rust index on leaf $(37.73 \%)$
$=$ followed by PR-2006-14, Divya and Kranti (35.26\%) in unprotected plots during 2012-13. (Table 1).

At 90 DAS, during 2011-12, maximum white rust index was recorded in genotype PR-2006$14(57.33 \%)$ followed by PRB-2008-5 and PRE-2007-6 (52.24\%) in unprotected plots. White rust index was significantly less in genotype PRB-2008-5 and PRE-2007-6(0.00) as compared to rest of the genotypes (13.62 $\%$ ) in protected plots.During 2012-13, white rust disease index on leaves was significantly less in genotype PRB-2008-5, PRE-2009-12, PRB-2004-3-4, Divya, NDRE-4 and Kranti (24.09\%) as compared to PRE-2007-6, PR2008-13, and PR-2006-14(25.33\%) in protected plots. Maximum white rust index was recorded in genotype NDRE-4(37.73\%) followed by PR-2006-14, Divya and Kranti $(35.26 \%)$ in unprotected plots (Table 1 ).

At 100 DAS, during 2011-12, maximum white rust index was recorded in genotype PR-2006$14(59.98 \%)$ followed by PRB-2008-5 and Kranti $(57.33 \%)$ in unprotected plots. White rust index was significantly less in genotype PR-2008-13 and NDRE-4(0.00) as compared to rest of the genotypes $(13.62 \%)$ in protected plots.

During 2012-13, white rust disease index on leaves was significantly less in genotypePRB2004-3-4(24.09\%) as compared to PRB-20085 and PRE-2009-12(28.58\%) in protected plots. Maximum white rust index was recorded in genotype PR-2006-14and NDRE$4(43.80 \%)$ followed by Divya (40.19\%) in unprotected plots (Table 2).

At 110 DAS, during 2011-12, maximum white rust index was recorded in genotype PR-2006$14(65.88 \%)$ followed by PRB-2008-5 and PRE-2007-6 (62.89\%) in unprotected plots. White rust disease was completely checked in all the genotypes in protected plots. 
Table.1 Effect of alternate spraying of Ridomil MZ and Mancozeb on White rust disease of Brassica juncea

\begin{tabular}{|c|c|c|c|c|c|c|c|c|c|c|c|c|c|c|c|c|}
\hline & \multicolumn{16}{|c|}{ White rust disease index (\%) } \\
\hline \multirow[t]{3}{*}{ Varieties } & \multicolumn{8}{|c|}{ 2011-12 } & \multicolumn{8}{|c|}{ 2012-13 } \\
\hline & \multicolumn{4}{|c|}{80 DAS } & \multicolumn{4}{|c|}{90 DAS } & \multicolumn{4}{|c|}{80 DAS } & \multicolumn{4}{|c|}{90 DAS } \\
\hline & $\mathbf{S}$ & US & Mean & DC & $\mathbf{S}$ & US & Mean & DC & $\mathbf{S}$ & US & Mean & DC & $\mathbf{S}$ & US & Mean & DC \\
\hline \multirow[t]{2}{*}{ PRB-2008-5 } & 8.33 & 54.15 & 31.32 & 84.62 & 8.33 & 62.48 & 35.49 & 86.67 & 0.00 & 16.66 & 8.33 & 100.00 & 16.66 & 22.49 & 19.66 & 25.93 \\
\hline & (13.62) & $(47.38)$ & $(30.50)$ & $(71.25)$ & $(13.62)$ & $(52.24)$ & $(32.93)$ & (73.93) & $(0.00)$ & $(24.09)$ & (12.04) & $(100.00)$ & (24.09) & $(28.29)$ & $(26.19)$ & $(14.84)$ \\
\hline \multirow[t]{2}{*}{ PRE-2009-12 } & 8.33 & 54.15 & 31.32 & 84.62 & 8.33 & 58.31 & 33.32 & 85.71 & 0.00 & 16.66 & 8.33 & 100.00 & 16.66 & 29.16 & 22.99 & 42.86 \\
\hline & $(13.62)$ & $(47.38)$ & $(30.50)$ & $(71.25)$ & $(13.62)$ & $(49.78)$ & $(31.70)$ & (72.64) & $(0.00)$ & $(24.09)$ & (12.04) & $(100.00)$ & $(24.09)$ & (32.64) & $(28.37)$ & $(26.21)$ \\
\hline \multirow[t]{2}{*}{ PRB-2004-3-4 } & 8.33 & 49.98 & 29.16 & 83.33 & 8.33 & 49.98 & 29.16 & 83.33 & 0.00 & 16.66 & 8.33 & 100.00 & 16.66 & 24.99 & 20.83 & 33.33 \\
\hline & (13.62) & (44.99) & $(29.31)$ & (69.72) & (13.62) & (44.99) & (29.31) & (69.72) & $(0.00)$ & (24.09) & (12.04) & (100.00) & (24.09) & (29.99) & (27.04) & (19.68) \\
\hline PRE-2007-6 & (13.62) & (47.38) & $(30.50)$ & (71.25) & (13.62) & (52.24) & (32.93) & (73.93) & (14.35) & (24.09) & (19.22) & $(40.45)$ & $(25.33)$ & (27.08) & $(26.21)$ & $(6.45)$ \\
\hline \multirow[t]{2}{*}{ PR-2008-13 } & 0.00 & 45.82 & 22.99 & 100.00 & 0.00 & 62.48 & 31.32 & 100.00 & 12.50 & 16.66 & 14.66 & 25.00 & 18.33 & 29.16 & 23.82 & 37.14 \\
\hline & $(0.00)$ & (42.59) & (21.30) & (100.00) & $(0.00)$ & (52.24) & (26.12) & (100.00) & (20.52) & (24.09) & (22.31) & (14.80) & (25.33) & (32.64) & (28.99) & (22.40) \\
\hline \multirow[t]{2}{*}{ PR-2006-14 } & 8.33 & 58.31 & 33.32 & 85.71 & 8.33 & 70.81 & 39.65 & 88.24 & 0.00 & 16.66 & 8.33 & 100.00 & 18.33 & 33.32 & 25.82 & 45.00 \\
\hline & (13.62) & (49.78) & (31.70) & (72.64) & (13.62) & (57.33) & (35.48) & (76.24) & $(0.00)$ & (24.09) & (12.04) & (100.00) & (25.33) & (35.26) & (30.29) & (28.15) \\
\hline \multirow{2}{*}{ Divya } & 8.33 & 45.82 & 27.16 & 81.82 & 8.33 & 54.15 & 31.32 & 84.62 & 12.50 & 16.66 & 14.66 & 25.00 & 16.66 & 33.32 & 24.99 & 50.00 \\
\hline & (13.62) & (42.55) & (28.08) & (67.98) & (13.62) & (47.38) & $(30.50)$ & (71.25) & (20.52) & (24.09) & (22.31) & (14.80) & (24.09) & (35.26) & (29.67) & (31.67) \\
\hline \multirow[t]{2}{*}{ NDRE-4 } & 0.00 & 41.65 & 20.83 & 100.00 & 0.00 & 37.49 & 18.83 & 100.00 & 12.50 & 16.66 & 14.66 & 25.00 & 16.66 & 37.49 & 27.16 & 55.56 \\
\hline & $(0.00)$ & (40.19) & (20.10) & (100.00) & $(0.00)$ & (37.73) & (18.87) & (100.00) & (20.52) & (24.09) & (22.31) & (14.80) & (24.09) & (37.73) & (30.91) & (36.16) \\
\hline Kranti & (13.62) & (44.99) & (29.31) & (69.72) & (13.62) & (52.24) & (32.93) & (73.93) & (20.52) & (24.09) & (22.31) & (14.80) & (24.09) & (35.26) & (29.67) & (31.67) \\
\hline \multirow[t]{2}{*}{ Mean } & 6.50 & 50.48 & 28.49 & 87.16 & 6.50 & 57.81 & 32.15 & 88.80 & 6.33 & 16.66 & 11.50 & 62.50 & 17.16 & 29.32 & 23.32 & 41.32 \\
\hline & (10.59) & (45.25) & (27.92) & (76.59) & (10.59) & (49.58) & $(30.09)$ & (78.63) & (10.72) & (24.09) & (17.40) & (55.52) & (24.50) & (32.68) & (28.59) & (25.03) \\
\hline C.D. $(5 \%)$ & 8.83 & 10.69 & & & 6.53 & 8.86 & & & 1.80 & 1.60 & & & 1.78 & 1.15 & & \\
\hline C.V. & 25.35 & 31.27 & & & 16.58 & 24.05 & & & 12.85 & 7.49 & & & 6.25 & 3.27 & & \\
\hline \multicolumn{17}{|l|}{ Protection } \\
\hline C.D. $(5 \%)$ & 3.22 & 4.32 & & & 3.02 & 4.33 & & & 1.16 & 1.04 & & & 1.42 & 0.95 & & \\
\hline C.V. & 19.81 & 27.04 & & & 16.44 & 25.16 & & & 17.67 & 10.48 & & & 10.70 & 5.83 & & \\
\hline \multicolumn{17}{|l|}{$\begin{array}{l}\text { Varieties X } \\
\text { Protection } \\
\end{array}$} \\
\hline C.D. (5\%) & 9.67 & 12.95 & & & 9.07 & 12.98 & & & 3.47 & 3.13 & & & 4.27 & 2.86 & & \\
\hline \multicolumn{17}{|l|}{$\begin{array}{l}\text { Protection X } \\
\text { Varieties } \\
\end{array}$} \\
\hline C.D. (5\%) & 11.17 & 14.07 & & & 9.15 & 12.76 & & & 3.04 & 2.73 & & & 3.51 & 2.32 & & \\
\hline
\end{tabular}

Note: Value in Parenthesis shows Angular transformation of real value 
Table.2 Effect of alternate spraying of Ridomil MZ and Mancozeb on White rust disease of Brassica juncea

\begin{tabular}{|c|c|c|c|c|c|c|c|c|c|c|c|c|c|c|c|c|}
\hline \multirow[t]{4}{*}{ Varieties } & \multicolumn{16}{|c|}{ White rust disease index (\%) } \\
\hline & \multicolumn{8}{|c|}{ 2011-12 } & \multicolumn{8}{|c|}{ 2012-13 } \\
\hline & \multicolumn{4}{|c|}{100 DAS } & \multicolumn{4}{|c|}{110 DAS } & \multicolumn{4}{|c|}{100 DAS } & \multicolumn{4}{|c|}{110 DAS } \\
\hline & $\mathbf{S}$ & US & Mean & DC & $\mathbf{S}$ & US & Mean & DC & $\mathbf{S}$ & US & Mean & DC & $\mathbf{S}$ & US & Mean & DC \\
\hline \multirow[t]{2}{*}{ PRB-2008-5 } & 8.33 & 70.81 & 39.65 & 88.24 & 0.00 & 79.14 & 39.65 & 100.00 & 22.99 & 31.32 & 27.16 & 26.67 & 33.32 & 52.15 & 42.65 & 36.00 \\
\hline & $(13.62)$ & $(57.33)$ & $(35.48)$ & $(76.24)$ & $(0.00)$ & $(62.89)$ & $(31.45)$ & $(100.00)$ & $(28.58)$ & $(33.97)$ & $(31.28)$ & $(15.87)$ & $(35.26)$ & $(46.18)$ & $(40.72)$ & $(23.66)$ \\
\hline \multirow[t]{2}{*}{ PRE-2009-12 } & 8.33 & 66.64 & 37.49 & 87.50 & 0.00 & 70.81 & 35.49 & 100.00 & 22.99 & 39.65 & 31.32 & 42.11 & 33.32 & 49.98 & 41.65 & 33.33 \\
\hline & $(13.62)$ & $(54.72)$ & $(34.17)$ & (75.11) & $(0.00)$ & $(57.33)$ & (28.67) & (100.00) & $(28.58)$ & (38.98) & (33.78) & (26.67) & $(35.26)$ & (44.99) & $(40.12)$ & (21.63) \\
\hline \multirow[t]{2}{*}{ PRB-2004-3-4 } & 8.33 & 58.31 & 33.32 & 85.71 & 0.00 & 66.64 & 33.32 & 100.00 & 16.66 & 33.32 & 24.99 & 50.00 & 33.32 & 49.98 & 41.65 & 33.33 \\
\hline & (13.62) & (49.78) & $(31.70)$ & (72.64) & $(0.00)$ & $(54.72)$ & (27.36) & (100.00) & (24.09) & $(35.26)$ & (29.67) & (31.67) & $(35.26)$ & (44.99) & $(40.12)$ & (21.63) \\
\hline \multirow[t]{2}{*}{ PRE-2007-6 } & 8.33 & 70.81 & 39.65 & 88.24 & 0.00 & 79.14 & 39.65 & 100.00 & 24.99 & 33.32 & 29.16 & 25.00 & 39.65 & 52.15 & 45.82 & 24.00 \\
\hline & (13.62) & (57.33) & $(35.48)$ & (76.24) & $(0.00)$ & $(62.89)$ & $(31.45)$ & (100.00) & (29.99) & $(35.26)$ & $(32.62)$ & (14.93) & (38.98) & $(46.18)$ & $(42.58)$ & (15.61) \\
\hline \multirow[t]{2}{*}{ PR-2008-13 } & 0.00 & 70.81 & 35.49 & 100.00 & 0.00 & 74.97 & 37.49 & 100.00 & 24.99 & 39.65 & 32.32 & 36.84 & 39.65 & 52.15 & 45.82 & 24.00 \\
\hline & $(0.00)$ & (57.33) & (28.67) & $(100.00)$ & $(0.00)$ & $(59.98)$ & (29.99) & $(100.00)$ & $(29.99)$ & $(38.98)$ & (34.48) & $(23.05)$ & (38.98) & $(46.18)$ & $(42.58)$ & $(15.61)$ \\
\hline \multirow[t]{2}{*}{ PR-2006-14 } & 8.33 & 74.97 & 41.65 & 88.89 & 0.00 & 83.30 & 41.65 & 100.00 & 24.99 & 47.98 & 36.49 & 47.83 & 33.32 & 56.31 & 44.82 & 40.74 \\
\hline & (13.62) & (59.98) & $(36.80)$ & $(77.29)$ & $(0.00)$ & (65.88) & (32.94) & $(100.00)$ & (29.99) & $(43.80)$ & (36.89) & $(31.52)$ & $(35.26)$ & $(48.58)$ & $(41.92)$ & (27.43) \\
\hline \multirow[t]{2}{*}{ Divya } & 8.33 & 62.48 & 35.49 & 86.67 & 0.00 & 70.81 & 35.49 & 100.00 & 24.99 & 41.65 & 33.32 & 40.00 & 33.32 & 52.15 & 42.65 & 36.00 \\
\hline & (13.62) & (52.24) & (32.93) & (73.93) & $(0.00)$ & (57.33) & (28.67) & (100.00) & (29.99) & (40.19) & (35.09) & (25.38) & $(35.26)$ & (46.18) & $(40.72)$ & (23.66) \\
\hline \multirow[t]{2}{*}{ NDRE-4 } & 0.00 & 45.82 & 22.99 & 100.00 & 0.00 & 54.15 & 27.16 & 100.00 & 24.99 & 47.98 & 36.49 & 47.83 & 33.32 & 56.31 & 44.82 & 40.74 \\
\hline & $(0.00)$ & (42.59) & (21.30) & (100.00) & $(0.00)$ & $(47.38)$ & (23.69) & (100.00) & (29.99) & $(43.80)$ & (36.89) & $(31.52)$ & $(35.26)$ & (48.58) & (41.92) & (27.43) \\
\hline \multirow[t]{2}{*}{ Kranti } & 8.33 & 70.81 & 39.65 & 88.24 & 0.00 & 74.97 & 37.49 & 100.00 & 20.83 & 37.49 & 29.16 & 44.44 & 33.32 & 41.65 & 37.49 & 20.00 \\
\hline & (13.62) & (57.33) & (35.48) & (76.24) & $(0.00)$ & $(59.98)$ & (29.99) & (100.00) & (27.08) & (37.73) & (32.41) & (28.24) & $(35.26)$ & (40.19) & $(37.72)$ & (12.28) \\
\hline \multirow[t]{2}{*}{ Mean } & 6.50 & 65.64 & 36.15 & 90.14 & 0.00 & 72.64 & 36.32 & 100.00 & 23.16 & 39.15 & 31.15 & 40.83 & 34.65 & 51.31 & 42.98 & 32.43 \\
\hline & (10.59) & $(54.29)$ & (32.44) & $(80.49)$ & $(0.00)$ & (58.71) & (29.36) & $(100.00)$ & $(28.70)$ & (38.66) & (33.68) & (25.77) & (36.08) & (45.79) & (40.93) & (21.19) \\
\hline C.D. $(5 \%)$ & 6.44 & 8.89 & & & 2.55 & 1.69 & & & 3.25 & 2.09 & & & 1.27 & 0.74 & & \\
\hline C.V. & 14.58 & 22.40 & & & 5.73 & 4.70 & & & 8.53 & 5.07 & & & 2.42 & 1.47 & & \\
\hline \multicolumn{17}{|l|}{ Protection } \\
\hline C.D. $(5 \%)$ & 3.07 & 4.35 & & & 1.25 & 0.82 & & & 0.49 & 0.29 & & & 0.97 & 0.56 & & \\
\hline C.V. & 14.90 & 23.46 & & & 6.04 & 4.88 & & & 2.73 & 1.50 & & & 3.95 & 2.40 & & \\
\hline \multicolumn{17}{|c|}{$\begin{array}{l}\text { Varieties X Protection } \\
\end{array}$} \\
\hline C.D. (5\%) & 9.22 & 13.06 & & & 3.76 & 2.46 & & & 1.46 & 0.87 & & & 2.99 & 1.69 & & \\
\hline \multicolumn{17}{|c|}{ Protection X Varieties } \\
\hline C.D. (5\%) & 9.17 & 12.82 & & & 3.69 & 2.42 & & & 3.41 & 2.18 & & & 2.42 & 1.40 & & \\
\hline
\end{tabular}

Note: Value in Parenthesis shows Angular transformation of real value 
Table.3 Effect of alternate spraying of Ridomil MZ and Mancozeb on Staghead incidence and severity of Brassica juncea

\begin{tabular}{|c|c|c|c|c|c|c|c|c|c|c|c|c|c|c|c|c|}
\hline \multirow[t]{4}{*}{ Varieties } & \multicolumn{16}{|c|}{ Staghead incidence \& Severity } \\
\hline & \multicolumn{8}{|c|}{ 2011-12 } & \multicolumn{8}{|c|}{ 2012-13 } \\
\hline & \multicolumn{4}{|c|}{ Disease incidence } & \multicolumn{4}{|c|}{ Disease severity } & \multicolumn{4}{|c|}{ Disease incidence } & \multicolumn{4}{|c|}{ Disease severity } \\
\hline & $\mathbf{S}$ & US & Mean & DC & $\mathbf{S}$ & US & Mean & DC & $\mathbf{S}$ & US & Mean & DC & $\mathbf{S}$ & US & Mean & DC \\
\hline \multirow[t]{2}{*}{ PRB-2008-5 } & 0.00 & 8.33 & 4.17 & 100 & 0.00 & 8.30 & 4.15 & 100 & 0.00 & 8.30 & 4.15 & 100 & 0.00 & 16.69 & 8.35 & 100 \\
\hline & $(0.00)$ & $(16.78)$ & $(8.39)$ & $(100.00)$ & $(0.00)$ & $(24.07)$ & $(12.03)$ & $(100.00)$ & $(0.00)$ & (16.74) & $(8.37)$ & $(100.00)$ & $(0.00)$ & $(26.91)$ & (13.46) & $(100.00)$ \\
\hline \multirow[t]{2}{*}{ PRE-2009-12 } & 0.00 & 12.84 & 6.42 & 100 & 0.00 & 13.11 & 6.55 & 100 & 0.00 & 13.11 & 6.55 & 100 & 0.00 & 37.37 & 18.69 & 100 \\
\hline & $(0.00)$ & (20.99) & $(10.50)$ & (100.00) & $(0.00)$ & (37.68) & (18.84) & (100.00) & $(0.00)$ & (21.22) & (10.61) & $(100.00)$ & $(0.00)$ & $(42.42)$ & (21.21) & (100.00) \\
\hline PRB-2004-3-4 & $(0.00)$ & (11.03) & $(5.52)$ & (100.00) & $(0.00)$ & (31.96) & (15.98) & (100.00) & $(0.00)$ & (11.15) & (5.57) & (100.00) & $(0.00)$ & (34.27) & (17.14) & (100.00) \\
\hline \multirow[t]{2}{*}{ PRE-2007-6 } & 0.00 & 14.42 & 7.21 & 100 & 0.00 & 15.05 & 7.52 & 100 & 0.00 & 15.05 & 7.52 & 100 & 0.00 & 22.34 & 11.17 & 100 \\
\hline & $(0.00)$ & $(22.32)$ & (11.16) & (100.00) & $(0.00)$ & (28.12) & (14.06) & (100.00) & $(0.00)$ & (22.82) & (11.41) & (100.00) & $(0.00)$ & (27.34) & (13.67) & (100.00) \\
\hline \multirow[t]{2}{*}{ PR-2008-13 } & 0.00 & 1.06 & 0.53 & 100 & 0.00 & 1.42 & 0.71 & 100 & 0.00 & 1.42 & 0.71 & 100 & 0.00 & 11.66 & 5.83 & 100 \\
\hline & $(0.00)$ & $(5.90)$ & (2.95) & (100.00) & $(0.00)$ & (19.88) & (9.94) & (100.00) & $(0.00)$ & $(6.83)$ & (3.42) & (100.00) & $(0.00)$ & (21.90) & (10.95) & (100.00) \\
\hline \multirow[t]{2}{*}{ PR-2006-14 } & 0.00 & 7.44 & 3.72 & 100 & 0.00 & 7.50 & 3.75 & 100 & 0.00 & 7.50 & 3.75 & 100 & 0.00 & 14.77 & 7.38 & 100 \\
\hline & $(0.00)$ & (15.83) & (7.91) & (100.00) & $(0.00)$ & (22.58) & (11.29) & (100.00) & $(0.00)$ & (15.89) & (7.94) & (100.00) & $(0.00)$ & (23.38) & (11.69) & (100.00) \\
\hline Divya & $(0.00)$ & $(5.74)$ & $(2.87)$ & (100.00) & $(0.00)$ & (17.46) & $(8.73)$ & $(100.00)$ & $(0.00)$ & $(5.87)$ & (2.94) & (100.00) & $(0.00)$ & $(19.00)$ & $(9.50)$ & $(100.00)$ \\
\hline \multirow[t]{2}{*}{ NDRE-4 } & 0.00 & 4.13 & 2.06 & 100 & 0.00 & 3.78 & 1.89 & 100 & 0.00 & 3.78 & 1.89 & 100 & 0.00 & 6.04 & 3.02 & 100 \\
\hline & $(0.00)$ & (11.72) & $(5.86)$ & (100.00) & $(0.00)$ & (14.18) & (7.09) & (100.00) & $(0.00)$ & (11.21) & (5.61) & (100.00) & $(0.00)$ & (14.83) & (7.42) & (100.00) \\
\hline \multirow[t]{2}{*}{ Kranti } & 0.00 & 4.70 & 2.35 & 100 & 0.00 & 4.61 & 2.30 & 100 & 0.00 & 4.61 & 2.30 & 100 & 0.00 & 14.30 & 7.15 & 100 \\
\hline & $(0.00)$ & (12.52) & $(6.26)$ & (100.00) & $(0.00)$ & $(22.20)$ & (11.10) & (100.00) & $(0.00)$ & (12.39) & $(6.20)$ & $(100.00)$ & $(0.00)$ & $(23.82)$ & (11.91) & (100.00) \\
\hline \multirow[t]{2}{*}{ Mean } & 0.00 & 6.40 & 3.20 & 100 & 0.00 & 6.50 & 3.25 & 100 & 0.00 & 6.50 & 3.25 & 100 & 0.00 & 17.81 & 8.90 & 100 \\
\hline & $(0.00)$ & (13.65) & $(6.82)$ & (100.00) & $(0.00)$ & (24.24) & (12.12) & (100.00) & $(0.00)$ & (13.79) & $(6.90)$ & (100.00) & $(0.00)$ & (25.99) & (12.99) & (100.00) \\
\hline C.D. $(5 \%)$ & 0.15 & 0.22 & & & 2.50 & 1.81 & & & 0.15 & 0.17 & & & 2.44 & 1.70 & & \\
\hline C.V. & 3.92 & 2.59 & & & 22.94 & 12.18 & & & 3.68 & 1.99 & & & 19.66 & 10.69 & & \\
\hline \multicolumn{17}{|l|}{ Protection } \\
\hline C.D. $(5 \%)$ & 0.08 & 0.11 & & & 1.17 & 0.85 & & & 0.07 & 0.08 & & & 1.10 & 0.79 & & \\
\hline C.V. & 4.26 & 2.76 & & & 23.05 & 12.26 & & & 3.63 & 2.01 & & & 19.06 & 10.58 & & \\
\hline \multicolumn{17}{|c|}{ Varieties X Protection } \\
\hline C.D. $(5 \%)$ & 0.23 & 0.32 & & & 3.52 & 2.55 & & & 0.20 & 0.24 & & & 3.31 & 2.36 & & \\
\hline \multicolumn{17}{|c|}{ Protection X Varieties } \\
\hline C.D. $(5 \%)$ & 0.23 & 0.31 & & & 3.53 & 2.55 & & & 0.20 & 0.24 & & & 3.38 & 2.38 & & \\
\hline
\end{tabular}
Note: Value in Parenthesis shows Angular transformation of real value 
White rust disease severity at leaf stage

\begin{tabular}{|l|l|}
\hline Rating score & Leaf area covered by the pustules $(\%)$ \\
\hline 0 & No symptoms \\
\hline 1 & $1-5$ \\
\hline 2 & $6-10$ \\
\hline 3 & $11-20$ \\
\hline 4 & $21-30$ \\
\hline 5 & $31-50$ \\
\hline
\end{tabular}

During 2012-13, white rust disease index on leaves was significantly less in genotype PRB-2008-5, PRE-2009-12 and PRB-2004-3$4(35.26 \%)$ as compared to PRE-2007-6 and PR-2008-13 (38.98\%) in protected plots. Maximum white rust index was recorded in genotype PR-2006-14 and NDRE-4 (48.58\%) followed by Divya (46.18\%) in unprotected plots. On an average the white rust severity on the leaves of protected plots was significantly less than that of unprotected plots (Table 2).

\section{Staghead incidence}

Staghead incidence (\% plant infected) was recorded at one week before maturity of Brassica juncea. The data revealed that all the genotypes were found to be free from staghead incidence under protected condition. During 2011-12, maximum staghead incidence was showed by genotype PRE2007-6 (22.32\%) followed by PRE-2009-12 (20.99\%).

Similarly, minimum staghead incidence was recorded in NDRE-4 (5.72\%) which was significantly lower than PR-2008-13 (5.90\%) under unprotected condition. During 2012-13, maximum staghead incidence was also found in genotype PRE-2007-6 (22.82\%) followed by PRE-2009-12 (21.22\%). Similarly, minimum staghead incidence was recorded in Divya $(5.87 \%)$ which was significantly lower than PR-2008-13 (6.83\%) under unprotected condition (Table 3 ).

\section{Staghead severity}

Staghead severity was also recorded one week before maturity of Brassica juncea for consecutive two years. The data revealed that all the genotypes were found to be free from staghead severity during 2011-12 and 201213 under protected condition. During 201112 , genotype NDRE-4 showed minimum staghead severity $(14.18 \%)$ followed by DIVYA (17.46\%) under unprotected condition. Similarly, maximum staghead severity was observed in genotype PRE-2009$12(37.68 \%)$ followed by PRB-2004-3-4 (31.96\%) under unprotected condition (Table $3)$.

During 2012-13, genotype NDRE-4 showed minimum staghead severity (14.83\%) followed by Divya (19.00\%) under unprotected condition. Similarly, maximum staghead severity was observed in genotype PRE-2009-12 (42.42\%) followed by PRB2004-3-4 (34.27\%) under unprotected condition (Table 3 ).

On an average White rust severity, staghead incidence and staghead severity was found significantly lower in protected plots as compare to unprotected plots hence there was significant reduction in disease severity have been noticed under protected plots. Maximum disease control (\%) up to $100 \%$ have been noticed under protected plots hence alternate sprays of Ridomil MZ and Mancozeb were 
found to be very effective in controlling White rust disease as well as staghead severity. Similar results were reported by Srivastava and Verma (1989), Mehta et al., (1996) and Kumar (1996). The effect of Ridomil MZ in the control of White rust and Downy mildew disease was reported by Kolte (1985).

\section{References}

Bains, S. S. and Jhooty, J. S. 1979.Mixed infections by Albugo candida and Hyaloperonospora parasitica on Brassica juncea inflorescence and their control. Indian Phytopathol. 32(2): 268271.

Bisht, I.S., Agrawal, R.C. and Singh, R. 1994. White rust (Albugo candida) severity in mustard (B. Juncea) varieties and its effects on seed yield. Plant Varieties and Seeds.7: 85-89.

Conn, K.L., Tiwari, J.P. and Awasthi, R.P. 1990.A disease assessment key for Alternaria black spot in rapeseed and mustard. Canadian Plant Dis. Survey, 70: $19-22$

GOI (Government of India). 2011. InAgricultural Statistics at a Glance. Agricultural Statistics Division, Dept. Agri. \& Coop., Ministry Agriculture, GOI, New Delhi

Kolte, S.J. 1985a. Disease management strategies for rapeseed mustard crops in India. Agric. Rev. 6: 81-88.

Kolte, S.J. 1985b. Diseases of Annual Edible Oilseed Crop.Vol.2 Rapeseed mustard and sesame diseases C.R.C. Press, Boca Raton, Florida, U.S.A.
Lakhra BS, Saharan GS.1991. Influence of thermo hydro and potential evapotranspiration on white rust epidemic of mustard. Cruciferae Newsletter, 14-15: 150-151.

Mehta, N., Saharan, G.S. and Kaushik, C.D. 1996. Efficacy and economics of fungicidal management of white rust and downy mildew complex in rapeseed-mustard. Indian J. Mycol. Plant Pathol. 26: 243-247.

Petrie, G.A. and Vanterpool, T.C. 1974. Fungi associated with hypertrophies caused by infection of cruciferae by Albugo candida cruciferarum. Can. Plant. Dis. Surv. 54: 37-42.

Saharan GS, Kaushik CD, Gupta PP, Tripathi NN.1984. Assessment of losses and control of white rust of mustard. Indian Phytopath37:397.

Saharan, G.S. 1992.Management of rapeseed and mustard diseases. In: Advances in oilseed research. D. Kumar and M. Rai (eds) sci. Pub., Jodhpur, India. 1 (7):152-188.

Sangeetha CG, Siddaramaiah AL (2007). Epidemiological studies of white rust, downy mildew and Alternaria blight of Indian mustard (Brassica juncea (Linn.) Czern. and Coss.) Afr. J. Agric. Res. 2(7): 305-308.

Srivastava, L. S. and Verma, R. N. 1987. Field screening of mustard cultivars against white rust in Sikkim. Indian $J$. Mycol. Pl. Path. 17(1): 46.

Wheeler BEJ (1969). An Introduction to Plant Diseases, John Wiley and Sons Limited, London, Pp. 301.

\section{How to cite this article:}

Ram, L., and Awasthi, R.P. 2018. Comparative Assessment of White Rust Disease in Improved Varieties of Indian Mustard. Int.J.Curr.Microbiol.App.Sci. 7(05): 1431-1438. doi: https://doi.org/10.20546/ijcmas.2018.705.170 